\title{
Three-dimensional thoracic aorta principal strain analysis from routine ECG-gated computerized tomography: feasibility in patients undergoing transcatheter aortic valve replacement
}

\author{
Alessandro Satriano ${ }^{1,2}$ (D) Zachary Guenther ${ }^{1,3}$, James A. White ${ }^{1,2}$, Naeem Merchant ${ }^{1,3}$, Elena S. Di Martino ${ }^{4}$, \\ Faisal Al-Qoofi ${ }^{2}$, Carmen P. Lydell ${ }^{1,3+}$ and Nowell M. Fine ${ }^{2^{*+}}$
}

\begin{abstract}
Background: Functional impairment of the aorta is a recognized complication of aortic and aortic valve disease. Aortic strain measurement provides effective quantification of mechanical aortic function, and 3-dimenional (3D) approaches may be desirable for serial evaluation. Computerized tomographic angiography (CTA) is routinely performed for various clinical indications, and offers the unique potential to study 3D aortic deformation. We sought to investigate the feasibility of performing 3D aortic strain analysis in a candidate population of patients undergoing transcatheter aortic valve replacement (TAVR).
\end{abstract}

Methods: Twenty-one patients with severe aortic valve stenosis (AS) referred for TAVR underwent ECG-gated CTA and echocardiography. CTA images were analyzed using a 3D feature-tracking based technique to construct a dynamic aortic mesh model to perform peak principal strain amplitude (PPSA) analysis. Segmental strain values were correlated against clinical, hemodynamic and echocardiographic variables. Reproducibility analysis was performed.

Results: The mean patient age was $81 \pm 6$ years. Mean left ventricular ejection fraction was $52 \pm 14 \%$, aortic valve area (AVA) $0.6 \pm 0.3 \mathrm{~cm}^{2}$ and mean AS pressure gradient (MG) $44 \pm 11 \mathrm{mmHg}$. CTA-based 3D PPSA analysis was feasible in all subjects. Mean PPSA values for the global thoracic aorta, ascending aorta, aortic arch and descending aorta segments were $6.5 \pm 3.0,10.2 \pm 6.0,6.1 \pm 2.9$ and $3.3 \pm 1.7 \%$, respectively. 3D PSSA values demonstrated significantly more impairment with measures of worsening AS severity, including AVA and MG for the global thoracic aorta and ascending segment $(p<0.001$ for all). 3D PSSA was independently associated with AVA by multivariable modelling. Coefficients of variation for intra- and inter-observer variability were 5.8 and $7.2 \%$, respectively.

Conclusions: Three-dimensional aortic PPSA analysis is clinically feasible from routine ECG-gated CTA. Appropriate reductions in PSSA were identified with increasing AS hemodynamic severity. Expanded study of 3D aortic PSSA for patients with various forms of aortic disease is warranted.

Keywords: Computerized tomography, Strain, 3-dimensional, Aortic valve stenosis, Transcatheter aortic valve replacement

\footnotetext{
* Correspondence: nmfine@ucalgary.ca

${ }^{\dagger}$ Equal contributors

2Division of Cardiology, Department of Cardiac Sciences, Libin Cardiovascular Institute of Alberta, University of Calgary, South Health Campus, 4448 Front Street SE, Calgary, Alberta T3M 1M4, Canada

Full list of author information is available at the end of the article
}

(c) The Author(s). 2018 Open Access This article is distributed under the terms of the Creative Commons Attribution 4.0 International License (http://creativecommons.org/licenses/by/4.0/) which permits unrestricted use, distribution, and reproduction in any medium, provided you give appropriate credit to the original author(s) and the source, provide a link to the Creative Commons license, and indicate if changes were made. The Creative Commons Public Domain Dedication waiver (http://creativecommons.org/publicdomain/zero/1.0/) applies to the data made available in this article, unless otherwise stated. 


\section{Background}

Alterations in aortic biomechanical properties are recognized to occur with advancing age and in patients with primary and secondary forms of aortopathy $[1,2]$. The resultant impairment in aortic function is an important contributor to global cardiovascular performance [3-5], and has been shown to have prognostic implications in specific patient cohorts, such as those with calcific degenerative aortic valve stenosis (AS) [6-8]. Indeed, associations between AS and increased aortic stiffness have been reliably demonstrated, leading to a theory that aortic disease may contribute to both symptom burden and clinical outcomes in this population $[6,8,9]$. However, optimal methods for assessing thoracic aorta biomechanics remains uncertain.

ECG-gated, contrast-enhanced computerized tomographic angiography (CTA) offers unique advantages for the study of aortic deformation. With complete 3dimensional (3D) visualization at high isotropic spatial resolution, small displacements of the aortic wall can be resolved throughout the complex architecture. Multiphase reconstruction of such imaging has previously been used to derive 2-dimensional (2D) strain, [10-12]. A methodology exists to perform in-vivo 3D aortic strain analysis requiring a single segmentation at end-diastole, optically and automatically tracked in 3 dimensions throughout the cardiac cycle relying on a velocity-field reconstructed for the whole dataset by means imagebased feature tracking, described by Satriano et al. [13]. Another approach exists leveraging on segmentations performed throughout the cardiac cycle, described by Pasta et al. [14]. In this approach, the point cloud obtained for the end-diastolic aortic luminal surface is projected normally onto the surfaces reconstructed for other cardiac phases [14]. However, the application of the single-segmentation approach [13] specifically to multi-phase CTA is previously undescribed. The latter is of interest given an emerging desire to serially and reproducibly evaluate regional patterns of disease across specific clinical populations [15]. Furthermore, the 3D optical tracking throughout the cardiac cycle [13] allows reconstruction of the full displacement field of the mesh, beyond the displacement of each node perpendicularly to the mesh. This allows for a comprehensive quantification of deformation [13]. Facilitating such an ability to evaluate 3D deformation, Principal strain analysis has been identified as an ideal deformation measure as it endeavors to measure tissue expansion without requirement for a pre-established axis of reference, describing the dominant (or principal) direction which strain is occurring within the tissue. This establishes a geometryindependent marker that may better represent vessel wall fiber orientation [16].

Exploiting the combined advantages of ECG-gated CTA and principal strain analysis, this study aimed to assess both the clinical feasibility and reproducibility of performing 3D principal aortic strain analysis in a clinical referral population. Among patients referred for transcatheter aortic valve replacement (TAVR), a population recognized for their burden of aortic disease and routinely referred for ECG-gated CTA, we identify the capacity of this technique to visualize and quantify global values and regional patterns of aortic deformation. Internal validation of aortic strain measures against expected influences of AS severity was also explored.

\section{Methods \\ Study population}

Twenty-one consecutive patients with severe, symptomatic AS referred for TAVR were included [17]. All patients underwent pre-operative assessment with ECGgated CTA and transthoracic echocardiography prior to TAVR. Patients with atrial fibrillation, contraindications to receiving iodinated contrast dye, and those having prior aortic or cardiac surgery were excluded. Clinical and demographic data and systemic blood pressure (measured by brachial artery cuff sphygmomanometer) were obtained at the time of echocardiography. This study was reviewed and approved by the University of Calgary Research Ethics Board, and a waiver of consent was granted for access to patient health information due to the retrospective nature of the analysis.

\section{Computed tomography imaging protocol}

A routine pre-TAVR protocol CTA was performed with retrospective ECG gating (10 phases throughout the cardiac cycle) and dose modulation in all cases [18]. CT examinations were performed on two 64-slice CT scanners (Discovery C750 HD, GE Healthcare, Milwaukee Wisconsin). A volume of $80-100 \mathrm{~mL}$ of commercially available intravenous ioversol $320 \mathrm{mg} / \mathrm{mL}$ contrast was injected at $5 \mathrm{~mL} / \mathrm{sec}$ followed by $50 \mathrm{~mL}$ of normal saline. The CT scanner detector collimation width was 0.625 $\mathrm{mm}$, detector coverage was $40 \mathrm{~mm}$, and the slice thickness was $0.625 \mathrm{~mm}$. Gantry rotation time was $0.35 \mathrm{sec}$ and the scan pitch was 0.16-0.2 depending on heart rate. The tube voltage was fixed at $100 \mathrm{kVp}$ and $120 \mathrm{kVp}$ for patients with a body mass index of $\leq 30$ and $>30$, respectively. Maximum tube current ranged from 400 and 700 $\mathrm{mA}$ with ECG-gated dose modulation reducing the tube current to $20 \%$ maximum between $80-20 \%$ of the R-R interval. Images were reconstructed using a standard algorithm at $10 \%$ intervals throughout the cardiac cycle, with a slice thickness of $0.625 \mathrm{~mm}$. This protocol resulted in a mean radiation exposure of $13.0 \pm 1.7 \mathrm{mSv}$. Data sets were transferred offline to stand-alone workstations for further analysis. 


\section{CTA strain analysis}

Three-dimensional strain analysis was performed as previously described, using a single segmentation of the aorta [13]. Briefly, an active-contour based segmentation of the thoracic aorta was performed from an enddiastolic CTA image series according to the approach described by Caselles et al.,[19] and implemented within a commercially available software platform ITK-SNAP [20]. No resampling of the obtained contouring was performed. The resultant 3D mesh model of the aorta was down-sampled and smoothed with a volume preserving approach,[21] using the commercially available software Meshlab [22]. Within the custom-built Matlab-based software that was previously described and validated, a velocity field was determined displacing the mesh from every $n$-th phase to the $(n+1)$-th phase for every phase from a single end-diastolic segmentation,[13] and the mean peak systolic maximum principal strain for the region of interest was computed [13]. Strain calculations were performed for each mesh element and then resolved for both the global thoracic aorta, and for each of the following segmental regions: ascending aorta (sinotubular junction to the brachiocephalic artery), aortic arch (brachiocephalic artery to the left subclavian artery) and descending thoracic aorta (left subclavian artery to the level of the diaphragm) [23]. All measurements were calculated in the form of peak principal strain amplitude (PPSA), from the Green-Lagrange strain tensor. The choice of computing strain over computing displacement allows quantification of aortic deformation without this being affected by rigid motion. This measurement describes the dominant direction of tissue lengthening, and is therefore expressed as a positive value [16].

\section{Echocardiographic imaging protocol}

Echocardiography was performed using a standardized clinical protocol with an iE33 system (Philips Medical Systems, Andover, Maryland) and an S5-1 transducer (1-5 MHz). Measurements were made from an average of 3 cardiac cycles. Assessment of left ventricular (LV) geometry and function and aortic valve (AV) function were performed according to published guidelines [24, 25]. LV volumes and ejection fraction (EF) were measured using the biplane Simpson method, and volumes were indexed to body surface area (BSA). Peak AV pressure gradient was calculated as: $\left(4 \times\right.$ [peak AV velocity $\left.\left.{ }^{2}\right]\right)$ , while mean pressure gradient was calculated by tracing the velocity-time integral (VTI) of the systolic transvalvular continuous-wave Doppler imaging signal. The LV outflow tact (LVOT) diameter was measured $5 \mathrm{~mm}$ proximal to the AV annulus. LV stroke volume was calculated according to the continuity equation as: (LVOT diameter $)^{2} \times 0.785 \times($ LVOT VTI $)$, and indexed to BSA, while the AV area was calculated as: (LVOT diameter) ${ }^{2}$ $\times 0.785 \times($ LVOT VTI/AV VTI $)$. The presence and severity of aortic valve regurgitation (AR) was graded based on multiple parameters as previously described [26]. Systemic vascular resistance was calculated as: $80 \times$ mean arterial pressure (MAP)/cardiac output (CO), where MAP is the diastolic blood pressure + (pulse pressure/3) and CO is the LV stroke volume $\times$ heart rate [27]. Systemic arterial compliance was calculated as LV stroke volume index/pulse pressure, and systemic arterial elastance as (systolic blood pressure $\times 0.9$ )/LV stroke volume index [8]. Valvulo-arterial impedance was calculated as: (systolic blood pressure + mean aortic valve pressure gradient)/LV stroke volume index, representing the valvular and arterial factors opposing LV ejection by absorption of LV mechanical energy [8].

\section{Statistical analysis}

Categorical variables are presented as counts with percentages, while continuous variables are expressed as the mean \pm standard deviation. Comparisons for continuous data were performed using 2-sample Student's $t$-test. Multiple comparisons of CTA-based 3D aortic PPSA between aortic segments were performed using one-way ANOVA. Relationships between 3D aortic PPSA and clinical, hemodynamic and echocardiographic parameters were evaluated using univariable linear regression analysis. A multivariable linear regression model was performed to assess the independent correlates of global thoracic aorta and ascending aorta 3D PPSA; including age, gender, history of hypertension, stroke, LVEF, AV area, and valvulo-arterial impedance. The amount of variance accounted for by these correlates was derived from the global $\mathrm{r}^{2}$ of the model. CTA-based 3D aortic PPSA intraobserver variability was evaluated by having one experienced observer perform the analysis on 10 randomly selected patients and then blindly repeating the analysis one week later. Interobserver variability assessment was conducted by having a second experienced and blinded observer perform the analysis on the same 10 patients. Intraobserver and interobserver variability testing was evaluated by Bland Altman analysis, and coefficients of variation were calculated. Additionally, both intra- and interobserver agreement were analyzed by calculating intra-class correlation (ICC) coefficient with 95\% confidence intervals (CI). All statistical analysis was performed using commercially available software (Matlab R2015b, The MathWorks, Inc., Natick, Massachusetts). A two-sided p-value of $\leq 0.05$ was considered statistically significant.

\section{Results}

Study subjects

Among the 21 patients studied, all had adequate CTA image quality for $3 \mathrm{D}$ aortic PPSA analysis. Baseline 
clinical characteristics are shown in Table 1. There was a high prevalence of hypertension, with a minority of patients having prior myocardial infarction, heart failure and stroke.

\section{Echocardiographic characteristics}

Baseline echocardiographic characteristics are presented in Table 2. The mean LVEF was $52.6 \pm 14.2 \%$. Five patients $(24 \%)$ had a reduced LVEF of $<45 \%$. Four patients (19\%) showed echocardiographic findings consistent with low-flow, low-gradient AS (defined as mean systolic transvalvular Doppler gradient $<40 \mathrm{mmHg}$, with concomitant LV stroke volume index $<35 \mathrm{~mL} / \mathrm{m}^{2}$ ) [28]. No patient had greater than mild aortic valve regurgitation. Significantly elevated systemic vascular resistance and arterial compliance, arterial elastance and valvuloarterial impedance were demonstrated. This is consistent with highly elevated valvular and arterial opposition to LV ejection coupled with a low stroke volume, characteristics consistent with chronic and severe AS.

Table 1 Baseline patient characteristics ( $N=21)$

\begin{tabular}{ll}
\hline Parameter & Value \\
\hline Clinical & \\
Age (years) & $81 \pm 6$ \\
Female, N (\%) & $6(29 \%)$ \\
Height (m) & $1.7 \pm 0.1$ \\
Weight (kg) & $80.7 \pm 17.2$ \\
Body mass index (kg/m²) & $24.8 \pm 4.1$ \\
Body surface area (m²) & $1.9 \pm 0.2$ \\
Hypertension, N (\%) & $12(57 \%)$ \\
Diabetes mellitus & $3(14 \%)$ \\
Hyperlipidemia & $10(48 \%)$ \\
Coronary artery disease, N (\%) & $12(57 \%)$ \\
Prior myocardial infarction, N (\%) & $6(29 \%)$ \\
Congestive heart failure, N (\%) & $8(38 \%)$ \\
Stroke, N (\%) & $3(14 \%)$ \\
Hemodynamic & \\
Heart rate (bpm) & $61 \pm 10$ \\
Systolic blood pressure (mmHg) & $124 \pm 16$ \\
Diastolic blood pressure (mmHg) & $63 \pm 12$ \\
Mean arterial pressure (mmHg) & $87 \pm 12$ \\
Pulse pressure (mmHg) & $56 \pm 14$ \\
Laboratory & $129 \pm 19$ \\
Creatinine (mcgmol/L) & $57 \pm 17$ \\
\hline Estimated glomerular filtration rate (mL/min) & \\
\hline
\end{tabular}

Data are expressed as mean \pm SD for continuous data and count (percentage) for categorical data
Table 2 Baseline echocardiographic characteristics $(N=21)$

\begin{tabular}{ll}
\hline Parameter & Value \\
\hline Left ventricular ejection fraction $(\%)$ & $52 \pm 14$ \\
Left ventricular end-diastolic volume index $\left(\mathrm{ml}^{\prime} / \mathrm{m}^{2}\right)$ & $53 \pm 16$ \\
Left ventricular end-systolic volume index $\left(\mathrm{ml} / \mathrm{m}^{2}\right)$ & $26 \pm 15$ \\
Left ventricular stroke volume index $\left(\mathrm{ml} / \mathrm{m}^{2}\right)$ & $26 \pm 7$ \\
Aortic valve annulus diameter $(\mathrm{mm})$ & $23 \pm 3$ \\
Aortic valve area $\left(\mathrm{cm}^{2}\right)$ & $0.6 \pm 0.3$ \\
Aortic valve mean pressure gradient $(\mathrm{mmHg})$ & $44 \pm 11$ \\
Aortic valve peak pressure gradient $(\mathrm{mmHg})$ & $76 \pm 18$ \\
Systemic vascular resistance $(\mathrm{mmHg} \mathrm{min} / \mathrm{L})$ & $28.9 \pm 8$ \\
Systemic arterial compliance $\left(\mathrm{ml} / \mathrm{mmHg} / \mathrm{m}^{2}\right)$ & $1.0 \pm 0.4$ \\
Systemic arterial elastance $(\mathrm{mmHg} / \mathrm{mL}))$ & $2.3 \pm 0.7$ \\
Valvulo-arterial impedance $\left(\mathrm{mmHg} / \mathrm{mL} / \mathrm{m}^{2}\right)$ & $6.7 \pm 2.1$
\end{tabular}

Data are expressed as mean \pm SD for continuous data and count (percentage) for categorical data. SD, standard deviation

\section{CTA 3D aortic strain}

Three-dimensional aortic PPSA values for the global thoracic aorta, and for the ascending aorta, aortic arch and descending aorta segments were $6.5 \pm 3.0,10.2 \pm 6.0$, $6.1 \pm 2.9$ and $3.3 \pm 1.7 \%$, respectively, with a statistically significant difference observed between each aortic segment $(\mathrm{p}<0.0001)$. An example of 3D aortic PPSA from a patient with AS awaiting TAVR is provided in Fig. 1 (Additional file 1), demonstrating the typical spatial distribution of 3D PPSA observed in this population with greater strain along the greater curve of the ascending aorta segment (consistent with AS jet directionality) followed by respective reductions in strain amplitude in the aortic arch and descending aorta segments. Fig. 1 demonstrates increased strain in the following regions 1) the ascending aorta, where the aortic valve outflow jet is present, 2) in proximity of the aortic branch vessels (where either a change in curvature or concave curvature is present), and 3) in regions of relatively greater curvature, such as the aortic arch, its branches, and regions of the descending aorta.

\section{Correlation of 3D aortic PSSA, patient characteristics and} echocardiographic measures

As reported in Table 3, neither global thoracic aortic 3D PPSA nor ascending aorta 3D PPSA were associated with any baseline clinical or hemodynamic variables in this patient population, inclusive of LV function, with the exception of prior stroke. In contrast, significant associations were observed between 3D aortic PPSA and echocardiographic measures of AS severity (Table 3). Specifically, significant correlations were observed for both global thoracic aorta and ascending aorta 3D PPSA measurements and AV area, and mean and peak AV pressure gradients, demonstrating more impaired strain 


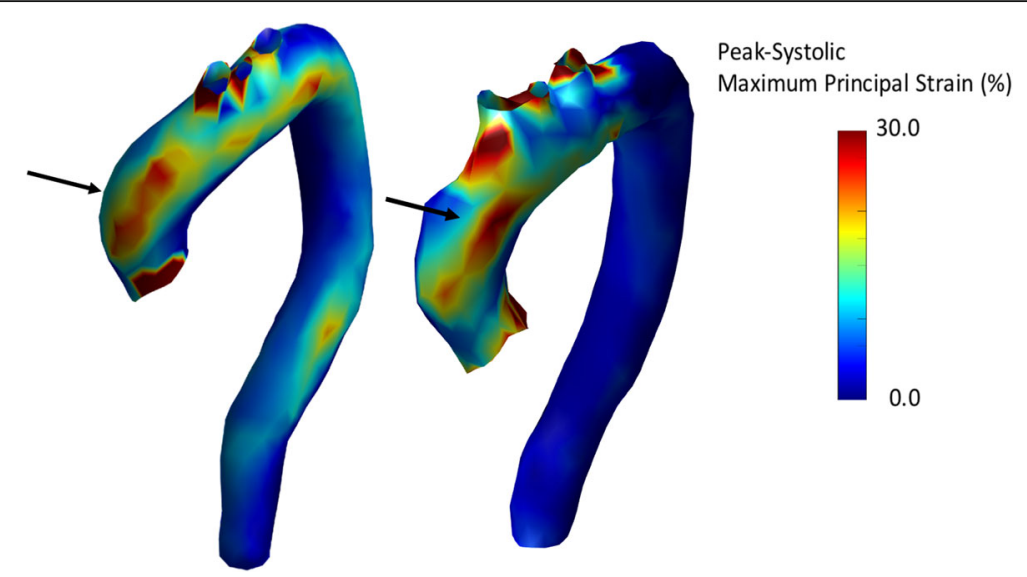

Fig. 1 Thoracic aorta 3-dimensional peak principal strain amplitude (PPSA) calculations using ECG-gated CTA from two patients with severe aortic valve stenosis awaiting transcatheter aortic valve replacement. These images demonstrate regional heterogeneity of 3D aortic PPSA with greater amplitude strain along the ascending aorta greater curvature (arrow, consistent with AS jet directionality) and relatively reduced strain amplitude in the aortic arch and descending aorta segments. The two patients demonstrate consistent regional strain patterns

with worsening AS severity. Linear regression plots describing this relationship between ascending aorta 3D PPSA and both AV area and mean AV gradient are presented in Fig. 2a and b, respectively. Corresponding plots for global thoracic aorta 3D PPSA are presented in Fig. 2c and d, respectively.

Multivariable linear regression analyses, performed separately for global thoracic aorta and ascending aorta 3D PPSA, and clinical, hemodynamic and echocardiographic variables are reported Table 4, and again demonstrate that measures of AS severity were the most significantly correlated to reduced aortic strain. The percentage of variance of 3D aortic PPSA accounted for by the variables included in the model are reported as the global $r^{2}$ (Table 4).

\section{Reproducibility}

Bland-Altman plots documenting high intra- and interobserver reproducibility for $3 \mathrm{D}$ global thoracic aorta PPSA are presented in Figs. 3 and 4, respectively. The coefficients of variation for intra- and inter-observer variability were 5.8 and $7.2 \%$, respectively. ICC values were significant for both intra- and interobserver reproducibility ( $p<0.0001$ for both). The intraobserver reproducibility value for 3D global thoracic aorta PSSA was 0 . 98 (0.92-0.99), while the interobserver reproducibility value was $0.96(0.86-0.99)$.

\section{Discussion}

This study demonstrates the clinical feasibility and reproducibility of 3D thoracic aorta wall principal strain mapping using routine ECG-gated CTA. Assessments of global and regional aortic biomechanics showed significantly greater strain impairment with worsening hemodynamic measures of AS severity, consistent with findings demonstrated previously by $2 \mathrm{D}$ aortic strain techniques [12, 29]. Accordingly, this study establishes the potential of routine ECG-gated CTA to provide reproducible measures of $3 \mathrm{D}$ global and regional aortic tissue health, and seeds interest for exploring such postprocessing techniques across a broad range of patients with aortic disease currently evaluated by CTA.

Multiple factors are recognized to contribute to increased aortic stiffness that are well expressed in our sentinel cohort of patients with AS, including advanced age, comorbidities such as hypertension, and underlying atherosclerotic disease that affects both the AV and the arterial vascular system $[9,30]$. The resultant impairment in thoracic aorta function has important biomechanical consequences on both ventricular performance and adverse remodeling, particularly among patients with AS due to impaired ventricular-arterial coupling given that both aortic stiffness and distensibility directly influence ventricular afterload incremental to that caused by valvular obstruction [3,31]. Previous studies have demonstrated the importance of assessing biomechanical thoracic aorta properties for the prediction of adverse cardiovascular outcomes, inclusive of heart failure and all-cause and cardiovascular mortality [3235]. Recently it has been demonstrated that vascular loading conditions may change dramatically with relief of valvular obstruction following TAVR, unmasking highly elevated aortic stiffness resulting in significant postprocedure hypertension [7]. Whether pre-procedure 3D thoracic aorta PPSA analysis incrementally improves prediction of similar adverse outcomes in this population requires further investigation. Our study found highly significant correlations between lower 3D thoracic aorta PPSA and worsening measures of AS severity. These findings suggest that the presence of severe AS has a 
Table 3 Correlations between 3-dimensional peak principal strain amplitude calculations using ECG-gated CTA of the ascending aorta and global thoracic aorta and clinical, hemodynamic and echocardiographic variables using linear regression analysis

\begin{tabular}{|c|c|c|c|c|}
\hline \multirow[b]{2}{*}{ Parameter } & \multicolumn{2}{|c|}{$\begin{array}{l}\text { Ascending } \\
\text { Aorta }\end{array}$} & \multicolumn{2}{|c|}{$\begin{array}{l}\text { Global Thoracic } \\
\text { Aorta }\end{array}$} \\
\hline & $\beta$ & $p$-value & $\beta$ & $p$-value \\
\hline \multicolumn{5}{|l|}{ Clinical } \\
\hline Age & -0.07 & 0.76 & 0.005 & 0.97 \\
\hline Gender & 2.43 & 0.41 & 0.59 & 0.69 \\
\hline Height & -0.23 & 0.13 & -0.09 & 0.2650 \\
\hline Weight & 0.04 & 0.61 & 0.02 & 0.68 \\
\hline Body mass index & 0.45 & 0.09 & 0.18 & 0.19 \\
\hline Body surface area & 1.04 & 0.86 & 0.57 & 0.84 \\
\hline Hypertension & 1.85 & 0.50 & -0.001 & 1.00 \\
\hline Diabetes mellitus & -2.88 & 0.45 & -2.20 & 0.24 \\
\hline Hyperlipidemia & 0.43 & 0.87 & 0.31 & 0.82 \\
\hline Coronary artery disease & -0.56 & 0.84 & -0.08 & 0.95 \\
\hline Prior myocardial infarction & 0.86 & 0.77 & 0.65 & 0.66 \\
\hline Congestive heart failure & -1.14 & 0.68 & 0.04 & 0.97 \\
\hline Stroke & 9.89 & 0.004 & 4.05 & 0.02 \\
\hline \multicolumn{5}{|l|}{ Hemodynamic } \\
\hline Heart rate & 0.009 & 0.94 & -0.005 & 0.93 \\
\hline Systolic blood pressure & 0.03 & 0.71 & 0.02 & 0.68 \\
\hline Diastolic blood pressure & -0.09 & 0.41 & -0.06 & 0.28 \\
\hline Mean arterial pressure & -0.05 & 0.69 & -0.03 & 0.59 \\
\hline Pulse pressure & 0.11 & 0.25 & 0.07 & 0.16 \\
\hline \multicolumn{5}{|l|}{ Echocardiographic } \\
\hline LV ejection fraction & 0.07 & 0.50 & 0.02 & 0.67 \\
\hline LV end-diastolic volume index & 0.001 & 0.99 & 0.03 & 0.41 \\
\hline LV end-systolic volume index & -0.002 & 0.98 & 0.03 & 0.49 \\
\hline AV annulus diameter & -0.08 & 0.85 & 0.17 & 0.43 \\
\hline AV area & 15.41 & 0.0005 & 8.54 & 0.00004 \\
\hline AV mean pressure gradient & -0.38 & 0.0004 & -0.19 & 0.0005 \\
\hline AV peak pressure gradient & -0.23 & 0.0003 & -0.11 & 0.0005 \\
\hline Systemic vascular resistance & -0.0001 & 0.49 & -0.001 & 0.30 \\
\hline Systemic arterial compliance & -2.29 & 0.55 & -0.97 & 0.61 \\
\hline Systemic arterial elastance & -0.64 & 0.73 & -0.61 & 0.51 \\
\hline Valvulo-arterial impedance & 0.03 & 0.77 & 0.01 & 0.74 \\
\hline
\end{tabular}

$A V$, aortic valve; $L V$, left ventricle

dominant influence on aortic deformation and remodeling that may significantly outweigh concurrent physiologic factors, a relationship that has also been described in prior reports $[7,12,36]$.

Localized, 2D aortic distensibility has been previously evaluated using cardiovascular magnetic resonance imaging (CMR) by measuring the difference in maximal and minimal short-axis lumen area at different phases of the cardiac cycle [32]. Despite being a simple and therefore attractive approach, this technique is unable to account for changes in longitudinal or other directions of aortic deformation, and therefore may be less sensitive for detecting clinically important alterations in thoracic aortic function [37, 38]. Bell et al. measured proximal thoracic aorta strain using CMR in $\mathrm{N}=375$ subjects by constructing a longitudinal centerline that follows the aortic curvature in a 2D plane, applying intersecting perpendicular chords to characterize changes in axial diameter throughout the cardiac cycle [37]. They found that proximal aortic stiffness was over-estimated when measured using circumferential strain calculations assessed from cross-sectional imaging due to the latent effects of longitudinal strain, and that incorporation of longitudinal strain into aortic biomechanical assessment may be an important component of cardiovascular risk assessment. Our technique derives 3D PPSA and therefore is not subject to the limitations of single-plane assessment, incorporating the 3D properties of aortic deformation without the need for further correction. Principal strain analysis has been previously used to characterize the complex fibre interactions occurring during ventricular contraction, $[16]$ and this same approach may be beneficial for providing a more physiologic metric of thoracic aorta deformation in AS and other cardiovascular diseases.

Previous reports have investigated 2D speckle-tracking echocardiography-based strain calculations of the thoracic aorta, postulating that strain is an accurate marker of aortic stiffness [39, 40]. The association demonstrated between aortic strain and collagen content provides further support for its value as a surrogate marker of vascular stiffness [41]. Teixeira et al. reported 2D circumferential ascending aorta strain values corrected for blood pressure were highly correlated with stroke volume and valvulo-arterial impedance in AS patients, concluding that both vessel wall and flow properties influence strain values [39]. Despite being widely available, echocardiography remains subject to coverage limitations and acoustic artifact for thoracic aorta imaging, particularly for multi-planar or 3D acquisitions, which may present challenges for accuracy and reproducibility.

Expanding interest in the study of ECG-gated CTA for the evaluation of vascular deformation is related to its full anatomic coverage of the thoracic aorta coupled with its superior spatial resolution, providing a dynamic isotropic dataset appropriate for quantitative biomechanical assessments [12]. Previous studies have used CTA to calculate circumferential and longitudinal 2D thoracic aorta strain based upon differences in vessel caliber throughout the cardiac cycle [10, 11, 15, 37]. More recently, Mileto et al. demonstrated the feasibility of 

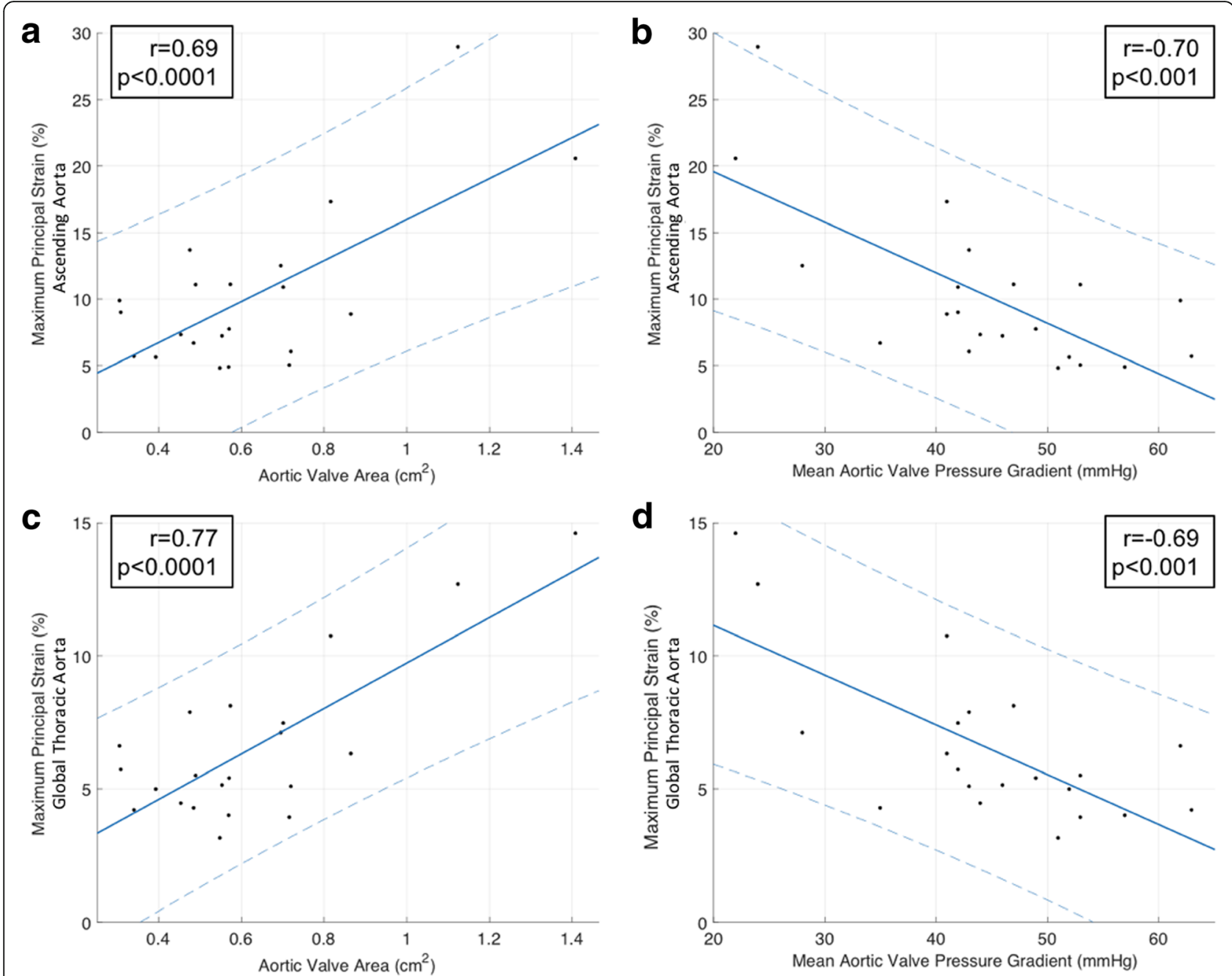

Fig. 2 Linear regression analysis demonstrating correlation between 3-dimensional (3D) thoracic aorta peak principal strain amplitude (PPSA) using ECG-gated CTA and measures of aortic valve stenosis severity. Correlations are depicted for the ascending aorta 3D PPSA and aortic valve area (a) and mean aortic valve pressure gradient (b), and for the global thoracic aorta 3D PPSA and aortic valve area (c) and mean aortic valve pressure gradient (d). Dotted lines indicate 95\% confidence intervals. R: Pearson Correlation Rank

performing aortic strain calculation at seven discrete locations using a deformable, motion-coherent modelling approach based on ECG-gated CTA acquisitions in a large cohort of patients $(\mathrm{N}=250)$ undergoing AV replacement [12]. Our technique offers a complimentary approach to provide a contiguous 3D principal strain analysis along the thoracic aortic. This provides a comprehensive spatial evaluation of deformation that is independent of aortic shape and orientation. We have identified regions of increased aortic wall strain in the aortic root and proximal ascending aorta that we speculate is secondary to the high velocity aortic valve outflow jet present in this population with severe AS referred for TAVR. Additionally, we have located high-strain regions in correspondence of the vessel branches, which are regions where curvature changes rapidly and can be concave. Our strain findings are consistent with previously described aortic regions of increased wall strain found in computer models [42, 43], ex-vivo optical strain measurements [44], and multi-segmentation based invivo strain analysis [14]. The need to quantify this inhomogeneity has been recently described [45]. Further, improved potential exists for inter-study registration given that a full volumetric evaluation is achieved, eliminating reliance on correct identification of specific anatomic landmarks [46].

\section{Limitations}

This study was a single-center feasibility study in a small number of subjects. Therefore, this technique requires validation in broader patient cohorts with a wider range of aortic disease. Due to the exposure to ionizing 
Table 4 Independent correlates of 3-dimensional peak principal strain amplitude calculations using ECG-gated CTA for the ascending aorta and global thoracic aorta

\begin{tabular}{|c|c|c|c|c|}
\hline \multirow[b]{2}{*}{ Parameter } & \multicolumn{2}{|c|}{ Ascending Aorta } & \multicolumn{2}{|c|}{ Global Thoracic Aorta } \\
\hline & $\bar{\beta}$ & $\overline{p \text {-value }}$ & $\bar{\beta}$ & $p$-value \\
\hline Age & -0.02 & 0.91 & 0.05 & 0.61 \\
\hline Gender & 3.05 & 0.15 & 0.89 & 0.37 \\
\hline Hypertension & 0.08 & 0.97 & -0.65 & 0.51 \\
\hline Stroke & 8.85 & 0.03 & 3.72 & 0.046 \\
\hline Heart rate & -0.04 & 0.75 & 0.003 & 0.95 \\
\hline Pulse pressure & -0.03 & 0.75 & 0.009 & 0.86 \\
\hline Left ventricular ejection fraction & -0.06 & 0.47 & -0.04 & 0.31 \\
\hline Aortic valve area & 14.41 & 0.008 & 3.73 & 0.003 \\
\hline Valvulo-arterial impedance & 0.02 & 0.80 & -0.004 & 0.93 \\
\hline Model-adjusted $r^{2}$ & 0.58 & 0.015 & 0.608 & 0.012 \\
\hline
\end{tabular}

The global $r^{2}$ of the model represents the amount of variance accounted for by these correlates

radiation and iodinated contrast dye required for this CTA-based technique, a control population was not recruited for comparison with the TAVR population in this analysis. Such comparison is an important consideration for future research into the clinical utility of this approach. However, future aims will include establishing reference values from broader registry data across a range of age, sex and comorbidity. Although such analyses were beyond the scope of this analysis, our approach was previously validated against ground-truth strain data obtained from a digital phantom [13]. Additionally, this study was not longitudinal, and therefore the prognostic value of aortic 3D strain analysis is uncertain. Further research is needed to determine the correlation between $3 \mathrm{D}$ aortic PSSA and clinical outcomes. Finally, we were unable to perform CTA and echocardiography in the same imaging session, therefore we cannot exclude the potential for altered loading conditions that may confound comparison of results between these two modalities.

\section{Conclusions}

Three-dimensional aortic wall principal strain analysis from routine, ECG-gated CTA is clinically feasible and shows good reproducibility. In this cohort of patients

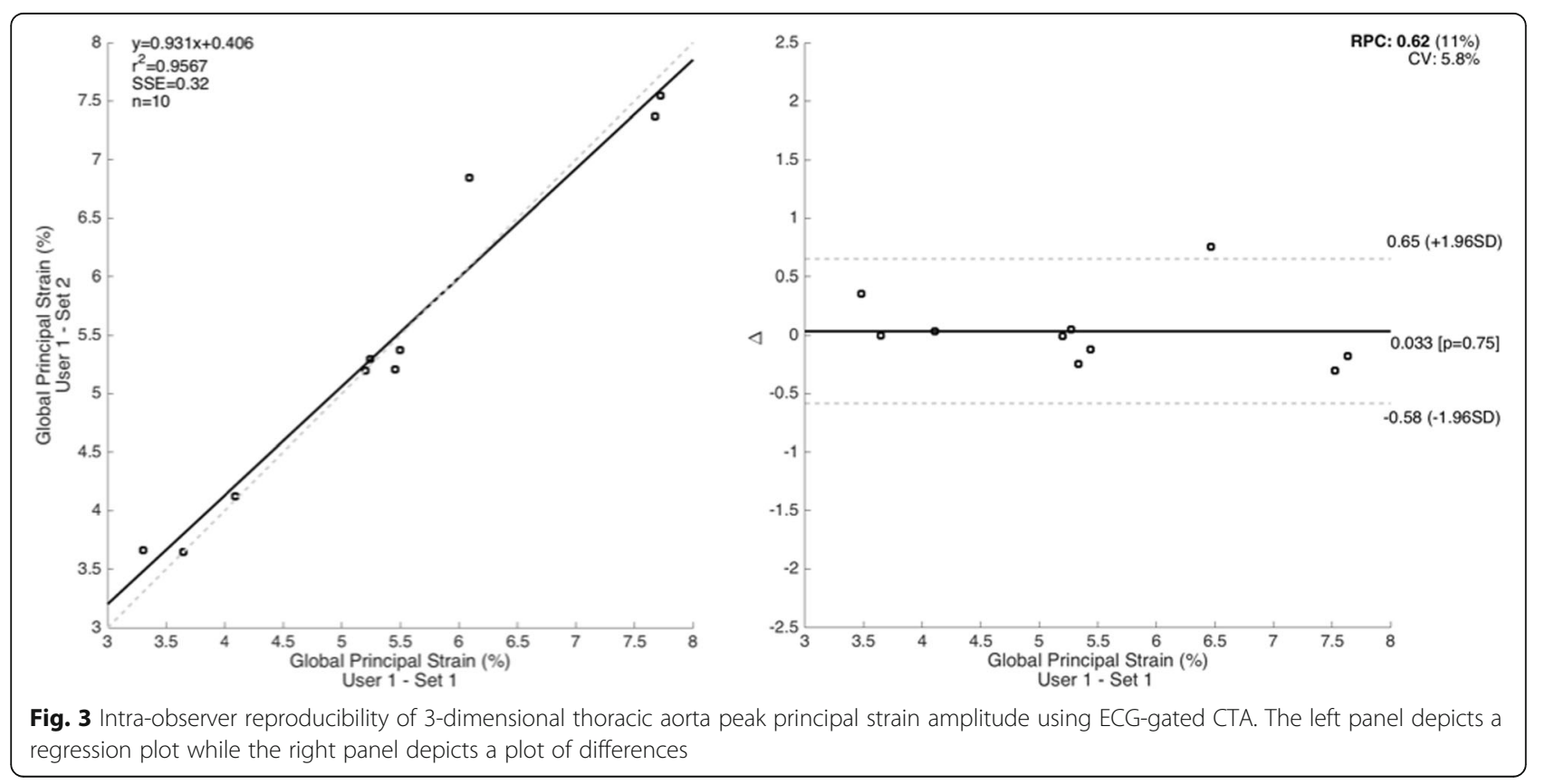



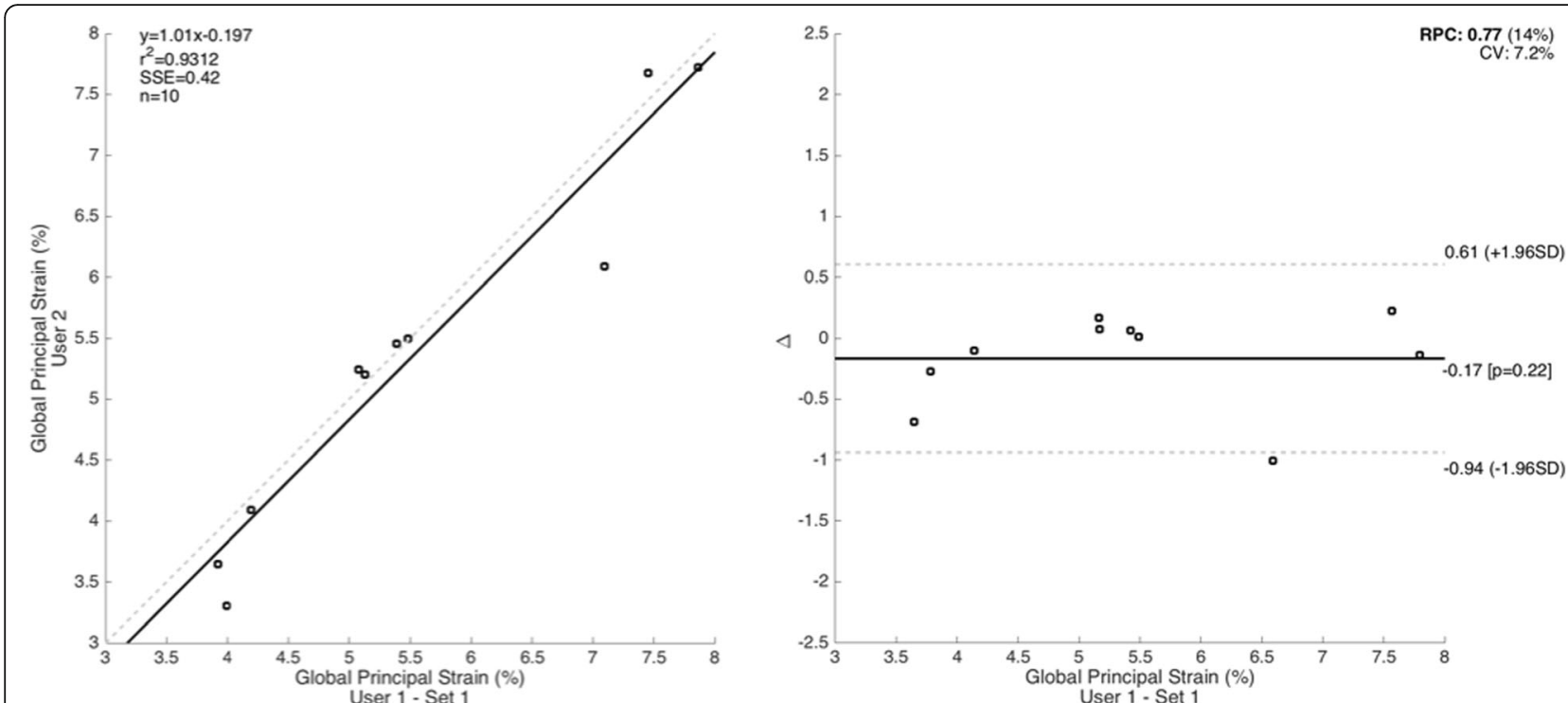

Fig. 4 Inter-observer reproducibility of 3-dimensional thoracic aorta peak principal strain amplitude using ECG-gated CTA. The left panel depicts a regression plot while the right panel depicts a plot of differences

with severe AS referred for TAVR, lower 3D PPSA values were appropriately identified in patients with greater hemodynamic severity of AS, as assessed by echocardiography. Future research is warranted to explore the value of this novel technique in other patient cohorts with aortic disease routinely imaged by ECGgated CTA.

\section{Additional file}

Additional file 1: Figure S1. Mesh and Corresponding CTA view throughout the cardiac cycle, for two separate subjects. For each patient, the upper panels depict the progression of the maximum principal strain distribution throughout the cardiac cycle. The lower panes depict the evolution of the aorta as seen in a CTA plane. (PNG $891 \mathrm{~kb}$ )

\section{Abbreviations}

2D: 2-dimensional; 3D: 3-dimensional; ANOVA: Analysis of variance; AR: Aortic valve regurgitation; AS: Aortic stenosis; AV: Aortic valve; AVA: Aortic valve area; BMI: Body mass index; BSA: Body surface area; CO: Cardiac output; CTA: Computerized tomography angiography; ECG: Electrocardiography; LV: Left ventricle; LVEF: Left ventricular ejection fraction; LVOT: Left ventricle outflow tract; MAP: Mean aortic pressure; MG: Mean gradient; PPSA: Peak principal strain amplitude; SD: Standard deviation; TAVR: Transcatheter aortic valve replacement; VTI: Velocity time integral

\section{Funding}

Dr. Satriano was funded by the Mitacs Elevate Postdoctoral Fellowship Program and by Medtronic of Canada, Ltd. Dr. White received research support from Circle Cardiovascular Inc. and is Chief Medical Officer of Cohesic Inc. Dr. White is supported by a Heart and Stroke Foundation of Alberta Early Investigator Award. This research was funded in part by the Calgary Health Trust.

\section{Availability of data and materials}

The datasets used and/or analysed during the current study are available from the corresponding author on reasonable request.

\section{Authors' contributions}

Conception and design: AS, ZG, JAW, FAQ, CPL, and NMF; analysis and interpretation of the data: AS, ZG, ESDM, NM, JAW and NMF; drafting of the article: AS, ZG, JAW, and NMF; revising of the article: AS, ZG, JAW, CPL, NM, ESDM, FAQ, and NMF. All authors have read and approved the final manuscript.

\section{Ethics approval and consent to participate}

This study was reviewed and approved by the University of Calgary Research Ethics Board, and a waiver of consent was granted for access to patient health information consistent with the conditions under section 50 of the Health Information Act of Alberta.

\section{Competing interests}

The authors declare that they have no competing interests.

\section{Publisher's Note}

Springer Nature remains neutral with regard to jurisdictional claims in published maps and institutional affiliations.

\section{Author details}

'Stephenson Cardiac Imaging Centre, University of Calgary, Calgary, Alberta, Canada. ${ }^{2}$ Division of Cardiology, Department of Cardiac Sciences, Libin Cardiovascular Institute of Alberta, University of Calgary, South Health Campus, 4448 Front Street SE, Calgary, Alberta T3M 1M4, Canada. ${ }^{3}$ Department of Diagnostic Imaging, Cummings School of Medicine, University of Calgary, Calgary, Alberta, Canada. ${ }^{4}$ Department of Civil Engineering and Centre for Bioengineering Research and Education, University of Calgary, Calgary, Alberta, Canada.

Received: 3 May 2017 Accepted: 24 April 2018

Published online: 02 May 2018

\section{References}

1. Lee H-Y, Oh B-H. Aging and arterial stiffness. Circ J. 2010;74(11):2257-62.

2. Sun Z. Aging, arterial stiffness, and hypertension. Hypertension. 2015;65(2): 252-6.

3. Laurent S, Cockcroft J, Van Bortel L, Boutouyrie P, Giannattasio C, Hayoz D, Pannier B, Vlachopoulos C, Wilkinson I, Struijker-Boudier H, et al. Expert consensus document on arterial stiffness: methodological issues and clinical applications. Eur Heart J. 2006;27(21):2588-605. 
4. Mancia G, De Backer G, Dominiczak A, Cifkova R, Fagard R, Germano G, Grassi G, Heagerty AM, Kjeldsen SE, Laurent S, et al. 2007 Guidelines for the Management of Arterial Hypertension: The Task Force for the Management of Arterial Hypertension of the European Society of Hypertension (ESH) and of the European Society of Cardiology (ESC). J Hypertens. 2007;25(6):1105-87.

5. Veerasamy M, Ford GA, Neely D, Bagnall A, MacGowan G, Das R, Kunadian V. Association of aging, arterial stiffness, and cardiovascular disease: a review. Cardiol Rev. 2014:22(5):223-32.

6. Hachicha Z, Dumesnil JG, Pibarot P. Usefulness of the valvuloarterial impedance to predict adverse outcome in asymptomatic aortic stenosis. J. Am. Coll. Cardiol. 2009:54(11):1003-11.

7. Yotti R, Bermejo J, Gutierrez-Ibanes E, Perez del Villar C, Mombiela T, Elizaga J, Benito Y, Gonzalez-Mansilla A, Barrio A, Rodriguez-Perez D, et al. Systemic vascular load in calcific degenerative aortic valve stenosis: insight from percutaneous valve replacement. J. Am. Coll. Cardiol. 2015;65(5):423-33.

8. Briand M, Dumesnil JG, Kadem L, Tongue AG, Rieu R, Garcia D, Pibarot P. Reduced systemic arterial compliance impacts significantly on left ventricular afterload and function in aortic stenosis: implications for diagnosis and treatment. J. Am. Coll. Cardiol. 2005;46(2):291-8.

9. Rosca M, Magne J, Calin A, Popescu BA, Pierard LA, Lancellotti P. Impact of aortic stiffness on left ventricular function and B-type natriuretic peptide release in severe aortic stenosis. Eur J Echocardiogr. 2011;12(11):850-6.

10. Morrison TM, Choi G, Zarins CK, Taylor CA. Circumferential and longitudinal cyclic strain of the human thoracic aorta: age-related changes. J Vasc Surg. 2009;49(4):1029-36.

11. Martin C, Sun W, Primiano C, McKay R, Elefteriades J. Age-dependent ascending aorta mechanics assessed through multiphase CT. Ann Biomed Eng. 2013:41(12):2565-74.

12. Mileto A, Heye TJ, Makar RA, Hurwitz LM, Marin D, Boll DT. Regional Mapping of Aortic Wall Stress by Using Deformable, Motion-coherent Modeling based on Electrocardiography-gated Multidetector CT Angiography: Feasibility Study. Radiology. 2016;280(1):230-6.

13. Satriano A, Rivolo S, Martufi G, Finol EA, Di Martino ES. In vivo strain assessment of the abdominal aortic aneurysm. J Biomech. 2015;48(2):354-60.

14. Pasta S, Agnese V, Di Giuseppe M, Gentile G, Raffa GM, Bellavia D, Pilato M. In Vivo Strain Analysis of Dilated Ascending Thoracic Aorta by ECG-Gated CT Angiographic Imaging. Ann Biomed Eng. 2017;45(12):2911-20.

15. Wittek A, Karatolios K, Fritzen CP, Bereiter-Hahn J, Schieffer B, Moosdorf R, Vogt $\mathrm{S}$, Blase C. Cyclic three-dimensional wall motion of the human ascending and abdominal aorta characterized by time-resolved three-dimensional ultrasound speckle tracking. Biomech Model Mechanobiol. 2016;15(5):1375-88.

16. Pedrizzetti G, Sengupta S, Caracciolo G, Park CS, Amaki M, Goliasch G, Narula J, Sengupta PP. Three-dimensional principal strain analysis for characterizing subclinical changes in left ventricular function. J Am Soc Echocardiogr. 2014;27(10):1041-50. e1041

17. Holmes DR Jr, Mack MJ, Kaul S, Agnihotri A, Alexander KP, Bailey SR, Calhoon JH, Carabello BA, Desai MY, Edwards FH, et al. 2012 ACCF/AATS/ SCAI/STS expert consensus document on transcatheter aortic valve replacement. J. Am. Coll. Cardiol. 2012;59(13):1200-54.

18. Freeman M, Webb JG, Willson AB, Wheeler M, Blanke P, Moss RR, Thompson CR, Munt B, Norgaard BL, Yang TH, et al. Multidetector CT predictors of prosthesis-patient mismatch in transcatheter aortic valve replacement. J Cardiovasc Comput Tomogr. 2013;7(4):248-55.

19. Caselles V, Catté F, Coll T, Dibos F. A geometric model for active contours in image processing. Numerische mathematik. 1993;66(1):1-31.

20. Yushkevich PA, Piven J, Hazlett HC, Smith RG, Ho S, Gee JC, Gerig G. Userguided $3 \mathrm{D}$ active contour segmentation of anatomical structures: significantly improved efficiency and reliability. Neuroimage. 2006;31(3):1116-28.

21. Taubin G. A signal processing approach to fair surface design. In: Proc 22nd Annu Conf Comput Graph Interact Tech: 1995; 1995. p. 351-8.

22. Cignoni P, Callieri M, Corsini M, Dellepiane M, Ganovelli G, Ranzuglia G. Meshlab: an open-source mesh processing tool. In: Eurographics Italian Chapter Conference; 2008. p. 129-36.

23. Goldstein SA, Evangelista A, Abbara S, Arai A, Asch FM, Badano LP, Bolen MA, Connolly HM, Cuellar-Calabria H, Czerny M, et al. Multimodality imaging of diseases of the thoracic aorta in adults: from the American Society of Echocardiography and the European Association of Cardiovascular Imaging: endorsed by the Society of Cardiovascular Computed Tomography and Society for Cardiovascular Magnetic Resonance. Journal of the American Society of Echocardiography : official publication of the American Society of Echocardiography. 2015;28(2):119-82.
24. Baumgartner H, Hung J, Bermejo J, Chambers JB, Evangelista A, Griffin BP, lung B, Otto CM, Pellikka PA, Quinones M, et al. Echocardiographic assessment of valve stenosis: EAE/ASE recommendations for clinical practice. Journal of the American Society of Echocardiography : official publication of the American Society of Echocardiography. 2009;22(1):1-23. quiz 101-102

25. Lang RM, Badano LP, Mor-Avi V, Afilalo J, Armstrong A, Ernande L, Flachskampf FA, Foster E, Goldstein SA, Kuznetsova T, et al. Recommendations for cardiac chamber quantification by echocardiography in adults: an update from the American Society of Echocardiography and the European Association of Cardiovascular Imaging. J Am Soc Echocardiogr. 2015;28(1):1-39. e14

26. Zoghbi WA, Enriquez-Sarano M, Foster E, Grayburn PA, Kraft CD, Levine RA, Nihoyannopoulos P, Otto CM, Quinones MA, Rakowski H, et al. Recommendations for evaluation of the severity of native valvular regurgitation with two-dimensional and Doppler echocardiography. Journal of the American Society of Echocardiography : official publication of the American Society of Echocardiography. 2003;16(7):777-802.

27. Kadem L, Dumesnil JG, Rieu R, Durand LG, Garcia D, Pibarot P. Impact of systemic hypertension on the assessment of aortic stenosis. Heart. 2005;91(3):354-61.

28. Hachicha Z, Dumesnil JG, Bogaty P, Pibarot P. Paradoxical low-flow, lowgradient severe aortic stenosis despite preserved ejection fraction is associated with higher afterload and reduced survival. Circulation. 2007; 115(22):2856-64.

29. Beller CJ, Labrosse MR, Thubrikar MJ, Robicsek F. Role of aortic root motion in the pathogenesis of aortic dissection. Circulation. 2004;109(6):763-9.

30. Allison MA, Cheung P, Criqui MH, Langer RD, Wright CM. Mitral and aortic annular calcification are highly associated with systemic calcified atherosclerosis. Circulation. 2006;113(6):861-6.

31. Ohyama Y, Ambale-Venkatesh B, Noda C, Chugh AR, Teixido-Tura G, Kim JY, Donekal S, Yoneyama K, Gjesdal O, Redheuil A, et al. Association of Aortic Stiffness With Left Ventricular Remodeling and Reduced Left Ventricular Function Measured by Magnetic Resonance Imaging: The Multi-Ethnic Study of Atherosclerosis. Circ. Cardiovasc. Imaging. 2016;9(7)

32. Redheuil A, Wu CO, Kachenoura N, Ohyama Y, Yan RT, Bertoni AG, Hundley GW, Duprez DA, Jacobs DR Jr, Daniels LB, et al. Proximal aortic distensibility is an independent predictor of all-cause mortality and incident CV events: the MESA study. J. Am. Coll. Cardiol. 2014;64(24):2619-29.

33. Redfield MM, Jacobsen SJ, Borlaug BA, Rodeheffer RJ, Kass DA. Age- and gender-related ventricular-vascular stiffening: a community-based study. Circulation. 2005;112(15):2254-62.

34. Hundley WG, Kitzman DW, Morgan TM, Hamilton CA, Darty SN, Stewart KP, Herrington DM, Link KM, Little WC. Cardiac cycle-dependent changes in aortic area and distensibility are reduced in older patients with isolated diastolic heart failure and correlate with exercise intolerance. J. Am. Coll. Cardiol. 2001;38(3):796-802.

35. Mitchell GF, Hwang SJ, Vasan RS, Larson MG, Pencina MJ, Hamburg NM, Vita JA, Levy D, Benjamin EJ. Arterial stiffness and cardiovascular events: the Framingham Heart Study. Circulation. 2010;121(4):505-11.

36. Harbaoui B, Courand PY, Girerd N, Lantelme P. Aortic Stiffness: Complex Evaluation But Major Prognostic Significance Before TAVR. J. Am. Coll. Cardiol. 2015;66(13):1521-2.

37. Bell V, Mitchell WA, Sigurethsson S, Westenberg JJ, Gotal JD, Torjesen AA, Aspelund T, Launer $\sqcup$, de Roos A, Gudnason V, et al. Longitudinal and circumferential strain of the proximal aorta. J. Am. Heart Assoc. 2014;3(6):e001536.

38. Weber TF, Muller T, Biesdorf A, Worz S, Rengier F, Heye T, Holland-Letz T, Rohr K, Kauczor HU, von Tengg-Kobligk H. True four-dimensional analysis of thoracic aortic displacement and distension using model-based segmentation of computed tomography angiography. Int J Cardiovasc Imaging. 2014;30(1):185-94.

39. Teixeira R, Moreira N, Baptista R, Barbosa A, Martins R, Castro G, Providencia L. Circumferential ascending aortic strain and aortic stenosis. Eur Heart J Cardiovasc Imaging. 2013;14(7):631-41.

40. Kim KH, Park JC, Yoon HJ, Yoon NS, Hong YJ, Park HW, Kim JH, Ahn Y, Jeong $\mathrm{MH}$, Cho JG, et al. Usefulness of aortic strain analysis by velocity vector imaging as a new echocardiographic measure of arterial stiffness. Journal of the American Society of Echocardiography : official publication of the American Society of Echocardiography. 2009;22(12):1382-8.

41. Kim SA, Lee KH, Won HY, Park S, Chung JH, Jang Y, Ha JW. Quantitative assessment of aortic elasticity with aging using velocityvector imaging and its histologic correlation. Arterioscler Thromb Vasc Biol. 2013;33(6):1306-12. 
42. Pasta S, Rinaudo A, Luca A, Pilato M, Scardulla C, Gleason TG, Vorp DA. Difference in hemodynamic and wall stress of ascending thoracic aortic aneurysms with bicuspid and tricuspid aortic valve. J Biomech. 2013;46(10): 1729-38.

43. Liang L, Liu M, Martin C, Sun W. A deep learning approach to estimate stress distribution: a fast and accurate surrogate of finite-element analysis. R Soc Interface. 2018;15(138)

44. Genovese K, Humphrey JD. Multimodal optical measurement in vitro of surface deformations and wall thickness of the pressurized aortic arch. J Biomed Opt. 2015;20(4):046005.

45. Ferraro M, Trachet B, Aslanidou L, Fehervary H, Segers P, Stergiopulos N. Should We Ignore What We Cannot Measure? How Non-Uniform Stretch, Non-Uniform Wall Thickness and Minor Side Branches Affect Computational Aortic Biomechanics in Mice. Ann Biomed Eng. 2018;46(1):159-70.

46. Csobay-Novak C, Fontanini DM, Szilagyi BR, Szeberin Z, Szilveszter BA, Maurovich-Horvat P, Huttl K, Sotonyi P. Thoracic aortic strain can affect endograft sizing in young patients. J Vasc Surg. 2015;62(6):1479-84.

Ready to submit your research? Choose BMC and benefit from:

- fast, convenient online submission

- thorough peer review by experienced researchers in your field

- rapid publication on acceptance

- support for research data, including large and complex data types

- gold Open Access which fosters wider collaboration and increased citations

- maximum visibility for your research: over $100 \mathrm{M}$ website views per year 